\title{
Uso didáctico de las TIC en las buenas prácticas de enseñanza de las matemáticas. Estudio de las opiniones y concepciones de docentes de educación secundaria en el departamento de Artigas
}

\author{
Didactic use of ICT within the good practices in Mathematics Teaching. \\ Study of the opinions and conceptions of Secondary School teachers in \\ the department of Artigas
}

ISSN 1510-2432 - ISSN 1688-9304 (en línea) - DOI: http://dx.doi.org/10.18861/cied.2015.6.2.34

Fabián Téliz

Master en Educación, Universidad ORT Uruguay. Profesor de Matemática, Centro Regional de Profesores del Litoral, Uruguay. Profesor de Matemática, Centro Regional de Profesores del Litoral e Instituto de Formación Docente de Artigas "María Orticochea”, Uruguay.

Fecha de recibido: $24 / 03 / 2015$

Fecha de aceptado: 31/05/2015

\section{Resumen}

El presente estudio abordó el uso didáctico de las Tecnologías de la Información y Comunicación (TIC) en las buenas prácticas de enseñanza de las matemáticas, particularmente en el departamento de Artigas, a partir del análisis de las opiniones y concepciones docentes. La temática resultó de interés teniendo en cuenta la extensión del Plan Ceibal a la educación secundaria así como la escasez de los trabajos de investigación sobre el impacto de las TIC en ese nivel.

La investigación se realizó desde un abordaje de triangulación metodológica que integró métodos cuantitativos y cualitativos. En primera instancia se aplicó una encuesta autoadministrada a todos los docentes de Matemática del departamento. A partir de ello, se seleccionó una muestra no probabilística de cinco docentes para profundizar el trabajo desde el abordaje cualitativo, empleando la entrevista en profundidad como principal herramienta. También se utilizó como técnica complementaria el análisis de documentos. A partir de la revisión de los estudios disponibles se ajustaron los instrumentos y, finalizada la recolección de datos se triangularon los resultados.

Los hallazgos revelaron que los profesores mantenían una actitud de disposición favorable ante la integración de las TIC en sus prácticas de enseñanza, destacándose aquellos ítems vinculados a la enseñanza frente a los asociados al aprendizaje. Sin embargo, el uso preferentemente instrumental (y limitado) de las TIC permitió apreciar que eran escasos los usos que de ellas realizaban los docentes desde una perspectiva constructivista para la enseñanza y el aprendizaje de las matemáticas, evidenciando la existencia mayoritaria de prácticas tradicionales de enseñanza.

Asimismo, se identificaron otros aspectos que condicionaban el uso o no uso de las TIC en las prácticas de enseñanza de las matemáticas. En general, estos factores estuvieron asociados a variables institucionales que inhibían la formación y el trabajo con las TIC que podían desarrollar los profesores, obstáculos que deberían sortearse para fomentar una real incorporación de las mismas a nivel institucional. 
Palabras claves: TIC, buenas prácticas de enseñanza, Matemática, Educación Secundaria.

\begin{abstract}
The present work is an approach to the didactic uses of ICT within the good practices in Mathematics Teaching -particularly in the department of Artigas- based on the analysis of teachers' reviews and conceptions. The topic has become relevant taking into consideration that the Plan Ceibal has extended to the sphere of Secondary Education and that little research has been carried out about the impact of ICT at this level.
\end{abstract}

The present research has been undertaken through a methodological triangulation approach including qualitative and quantitative methods. First of all, a self-administrated poll was applied to all Math teachers of the department of Artigas. For a deeper qualitative approach, a non probabilistic sample of five teachers was selected, with interview as the main technique. Document analysis was used as an additional technique. After reviewing the available studies, instruments were adjusted and upon completion of data recollection, the results were triangulated.

Findings revealed that teachers maintain a positive attitude toward integrating ICT into their teaching practices. Those items related to teaching prevailed over those related to learning. However, the instrumental -and limited- use of ICT shows that such technologies are not being applied from a constructivist perspective, evidencing, thus, the predominance of traditional teaching practices.

Other aspects that condition the use of ICT in the practice of Math teaching were identified. Such depended, in general, on institutional variables that inhibit teachers from training and working with ICT, obstacles that should be overcome in order to promote a real incorporation and integration of these technologies at an institutional level.

Key words: ICT, good teaching practices, Mathematics, Secondary School.

\title{
1. Introducción
}

Vivimos épocas de profundas transformaciones. Avanzamos, inexorablemente, hacia la "Sociedad de la Información y el Conocimiento" (Pozo, 2000), en la cual las TIC están cada vez más presentes en las actividades diarias de los ciudadanos, en consenso con Lugo y Kelly (2010:1) cuando afirman que dichas tecnologías "han venido para quedarse".

Como no podría ser diferente, la educación no es ajena a esta realidad quien exige a aquella respuestas planificadas, así como le exige considerar las que no se han previsto para responder a las nuevas demandas.

Estudios realizados a nivel nacional (Rivoir, 2009; Rodríguez Zidán et al., 2009; Ravela y Pérez Gomar, 2012) confirman que las respuestas de los docentes ante la incorporación de las TIC a sus prácticas de enseñanza todavía son escasas y, en muchos casos, de resistencia. Esta situación condujo al autor del presente trabajo a formularse algunos cuestionamientos sobre los cuales se construyó el siguiente problema de investigación: ¿Cuál es el perfil de uso didáctico de las TIC en las buenas prácticas de enseñanza de las matemáticas en la educación secundaria en el departamento de Artigas? ¿Cuáles son las concepciones docentes que favorecen u obstaculizan el desarrollo de las mismas?

Tejedor et al. (2009) señala que el uso de las TIC en las prácticas de enseñanza está condicionado por el dominio que del recurso posean los docentes, así como también por 
el potencial pedagógico que le atribuyan y por las actitudes que mantengan hacia dichas prácticas, entre otros factores.

Según López y Basto (2009), diversas investigaciones realizadas desde diferentes enfoques, y refiriéndose a las concepciones, creencias, constructos y teorías de los docentes coinciden en señalar "cómo estos subyacen en sus acciones pedagógicas y cómo gran parte de los cambios educativos son posibles si los agentes que participan en ellos están dispuestos a modificar sus creencias y sus prácticas".

En la literatura disponible, son variadas las definiciones acerca de qué es una "buena práctica"1 de enseñanza con TIC. En el contexto de este trabajo de investigación, se utiliza la definición de Sosa, Peligros y Díaz Muriel (2010:152), quienes señalan que es "toda aquella práctica educativa que con el uso de las TIC supone una mejora o potencialización del proceso de enseñanza-aprendizaje y por tanto de sus resultados, pudiendo servir, además, de referencia a otros contextos". Por tal motivo se sostiene, a modo de hipótesis, que las concepciones de los profesores en relación a la enseñanza de las matemáticas mediante TIC serían determinantes para su integración en dicho proceso, así como también condicionarían la forma de llevarlo a cabo.

Desde esa perspectiva, el objetivo general del presente trabajo fue comprender las relaciones entre las concepciones docentes sobre el uso didáctico de las TIC y las buenas prácticas de la enseñanza de las matemáticas mediadas por TIC en la educación secundaria del departamento de Artigas.

Como objetivos específicos, se formularon los siguientes:

- Describir el perfil de uso didáctico de las TIC en las prácticas de enseñanza de las matemáticas en educación secundaria.

- Identificar las opiniones y concepciones docentes sobre el uso didáctico de las TIC y sus valoraciones sobre las buenas prácticas de enseñanza con TIC en las aulas de matemáticas de educación secundaria.

- Indagar acerca de las expectativas docentes al desarrollar procesos de enseñanza con la mediación de las TIC en las aulas de matemáticas de educación secundaria.

- Analizar la coherencia entre las concepciones docentes sobre el uso didáctico de las TIC y sus propuestas de enseñanza mediadas por TIC, en las aulas de matemáticas de educación secundaria.

En la opinión del autor del presente trabajo de investigación, el mismo contribuyó a aportar nuevos elementos de análisis de esta política educativa de incorporación de TIC al sistema educativo uruguayo, desde un enfoque que centró su mirada en las concepciones del docente de matemáticas de educación secundaria, dejando de lado los factores administrativos u organizativos en que muchas veces se han centrado los estudios disponibles hasta el momento.

\section{Buenas prácticas de enseñanza de las matemáticas con tic}

Como señalan Pozo et al. (2006:34), "todos tenemos creencias o teorías profundamente asumidas y tal vez nunca discutidas, sobre lo que es aprender y enseñar, que rigen nuestras acciones al punto de construir un verdadero currículo oculto que guía, a veces sin nosotros saberlo, nuestra práctica educativa". 
Según lo constatado por los estudios TEDS-M (2012), las creencias sobre la disciplina, su aprendizaje y enseñanza condicionan las prácticas de los docentes. El enfoque desde el cual estos se sitúen para pensar acerca de la enseñanza de los contenidos matemáticos a través de las TIC, sin lugar a dudas lleva a la necesidad de fundamentar las decisiones en base a los aportes de las investigaciones en el campo de la Didáctica de la Matemática.

Según indica Castillo (2008:15), el Consejo Estadounidense de Profesores de Matemática (NCTM) establece, en general, una serie de pautas relativas a la educación matemática en la actualidad. En particular, con respecto a la tecnología afirma que "resulta esencial en la enseñanza y el aprendizaje, ya que influye en las matemáticas que se enseñan y mejoran el proceso de aprendizaje de los estudiantes", realzando el papel de las TIC para la mejora de los aprendizajes a partir de una "enseñanza efectiva"2 sustentada desde el paradigma constructivista.

En las posturas epistemológicas actuales de la Didáctica de la Matemática es posible identificar las diversas oportunidades que brindan las TIC para la realización de la "transposición didáctica" (Chevallard, 1991). En ese sentido, las mismas posibilitan el abordaje, de modo más dinámico y efectivo, de contenidos que antes resultaban complejos. El siguiente diagrama, tomado de Podestá (2011), permite apreciar cómo es posible conjugar en una misma práctica de enseñanza los lineamientos actuales de la Didáctica de la Matemática con las potencialidades que brinda la inclusión de las TIC.

Actividades que se espera que el alumno realice en el marco de una práctica de enseñanza según el modelo 1 a 1 y la Didáctica de la Matemática.

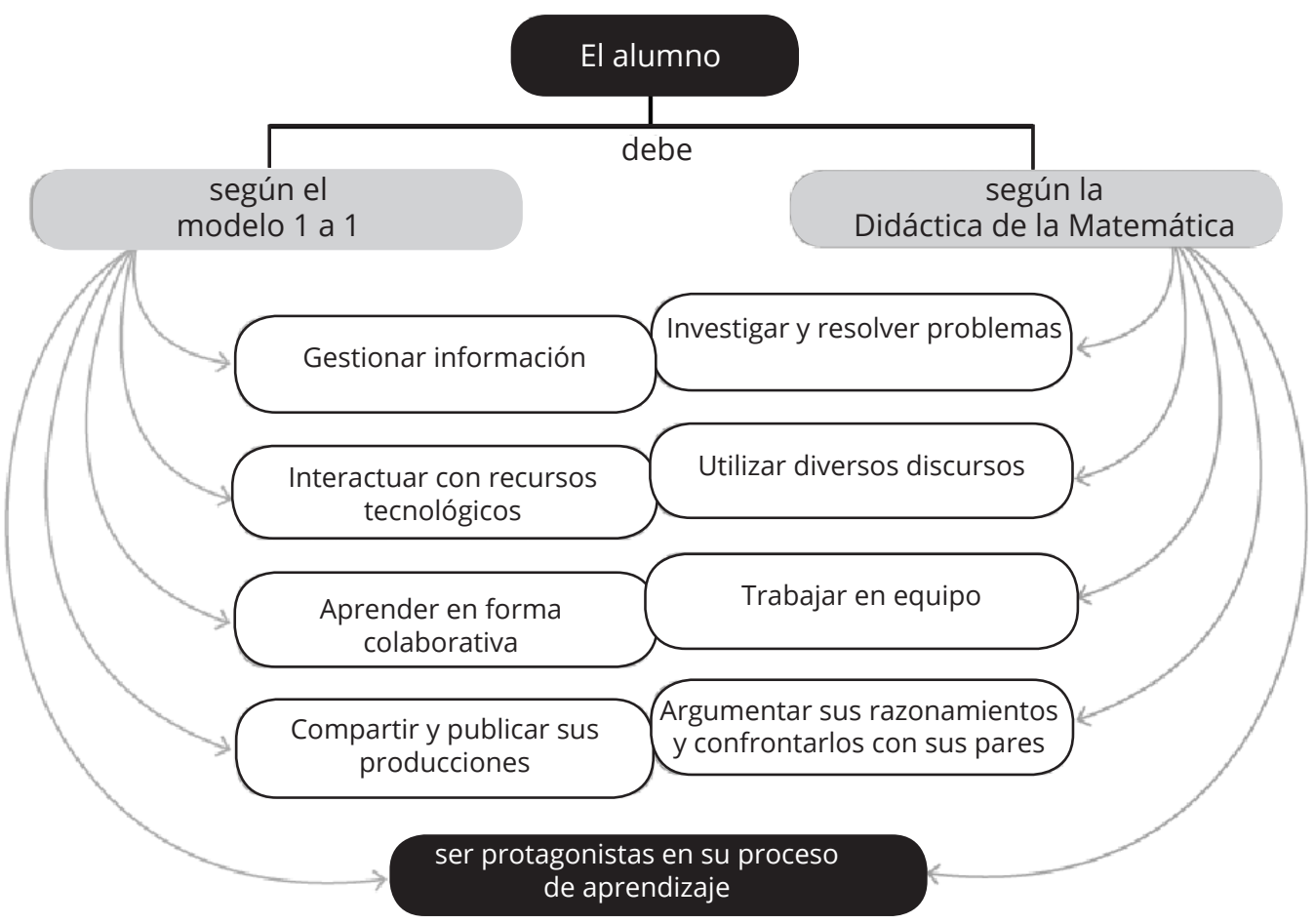


Desde esa perspectiva, que se caracteriza por diseñar situaciones de enseñanza que desafíen los conocimientos de los estudiantes permitiéndoles generar y construir conocimientos, es necesario que el docente desempeñe un nuevo rol que exige nuevas competencias y/o habilidades profesionales.

El principal reto está en lograr generar un equilibrio entre las concepciones y creencias ya arraigadas en el docente y la aplicación de nuevos enfoques o paradigmas didácticos alternativos, que permita conciliar los intereses de los estudiantes con las expectativas docentes, integrando las TIC como una herramienta que contribuya en ese sentido.

\section{Plan Ceibal y profesores de matemática}

En el año 2010, a partir de la llegada de los primeros egresados de Educación Primaria luego de la implementación del Plan Ceibal, el mismo comenzó a recorrer sus primeros pasos en Educación Media. Sin embargo, aún no existen grandes evaluaciones ni trabajos de investigación que analicen el impacto de este proyecto en ese nivel del sistema educativo.

El Departamento de Monitoreo y Evaluación de Plan Ceibal realizó, en 2011, una encuesta telefónica a docentes de Educación Media Pública. Los datos relevados específicamente con respecto a los docentes de matemáticas indican que en su gran mayoría dichos docentes disponen de computadoras en su hogar para su uso: entre los docentes que se desempeñan en el CES el porcentaje es de $99 \%$, disminuyendo a $96 \%$ con respecto a aquellos que se desempeñan en el CETP Sin embargo, al relevar la tenencia de computadoras XO o Magallanes, la situación se invierte: en el CES solamente el 2 \% de los encuestados cuenta con el recurso, mientras que entre los docentes del CETP, el porcentaje asciende a $8 \%$. Consultados sobre si disponen de conexión a Internet desde el hogar, el $3 \%$ de los docentes de cada subsistema manifiesta no contar con el servicio, aunque en el CETP hay un $2 \%$ adicional que se incluye en la categoría "NS/NC", lo que puede indicar ausencia del servicio en el hogar. (Ceibal, 2011b).

Consultados sobre la utilización de las computadoras para "dar clases" durante el año lectivo 2010, se observa que un $32 \%$ de los docentes que se desempeñan en el CES no las ha utilizado, lo que aumenta a $38 \%$ entre los docentes del CETP Aunque no se explicita en las conclusiones del informe, el autor del presente trabajo considera que esa situación se debe en gran medida a la falta de capacitación para un uso pedagógico del recurso en el aula. Preguntados acerca de “¿En qué áreas entiende usted que necesitará mayor capacitación?", los docentes de ambos subsistemas coinciden en señalar como prioridad "En estrategias didácticas para usar la computadora en el aula", indicado por el 80 \% de los docentes del CES y por el $77 \%$ de los docentes del CETP (Ceibal, 2011b).

La formación y capacitación de los docentes es una de las prioridades a atender, pues la clave del éxito radica en la capacidad del profesor para "crear, adaptar, presentar y compartir actividades didácticas innovadoras, mediadas por tecnología, donde ésta enriquece la práctica, genera interacción, facilita la adopción, flexibiliza los diferentes ritmos de aprendizaje y promueve genuinos cambios inspiradores que enriquecen el currículo" (Ceibal, 2011a:8).

Para finalizar, se consultó a los profesores sobre sus percepciones acerca del impacto del Plan Ceibal en diferentes aspectos y se les solicitó indicaran si creían que el aporte sería positivo, neutro o negativo. Los resultados presentados indican únicamente los porcentajes de docentes cuyas percepciones son positivas en cada caso. “Diversificación de los recursos 
didácticos disponibles", es el aspecto en el que los docentes perciben que el programa de innovación tendrá su mayor impacto (91\% para los docentes del CES y 90 \% para los docentes del CETP), seguido del "Acceso a información para los docentes y estudiantes" (87 $\%$ en ambos subsistemas). Por su parte, visualizan en una menor proporción el impacto positivo en la "organización del trabajo de aula del docente" (62 \% para los docentes del CES y $71 \%$ para los docentes del CETP). (Ceibal, 2011 b).

Asimismo, el Instituto de Educación de la Universidad ORT Uruguay ha desarrollado, en el marco de un llamado realizado por Plan Ceibal, un proyecto titulado "El Plan Ceibal y las prácticas de los docentes de Matemática en primer año de liceo", durante los años 2011 y 2012.

Según los datos relevados acerca del perfil de los profesores de Matemática de primer año de educación secundaria, $74 \%$ de los docentes son mujeres. Con respecto a la edad, tres de cada diez docentes tienen menos de veintinueve años. La alta rotación y la baja titulación de los docentes siguen siendo factores que se constatan en la población estudiada: solamente el $35 \%$ presenta título docente (Vaillant y Bernasconi, 2013:8).

Como se señala en un reciente trabajo (Rodríguez Zidán y Téliz, 2013), la tenencia de un título habilitante es una de las variables fundamentales para lograr niveles de calidad en la enseñanza. Los diferentes estudios y censos nacionales realizados en la órbita de la Administración Nacional de Educación Pública (ANEP) han demostrado que los docentes de Matemática de Educación Secundaria presentan un nivel de titulación muy bajo³.

En cuanto al "perfil de acceso y uso de TIC", los datos confirman lo constatado por la Encuesta Telefónica realizada por Plan Ceibal: el acceso a las computadoras desde el hogar es superior al que se realiza desde el centro educativo. Además, “Usan TIC para acceder a recursos informáticos e información en Internet y menos para intercambiar información con colegas". Respecto a sus percepciones sobre la evolución del uso de las TIC durante el año del estudio, el 42 \% de los docentes encuestados manifestó no haber evolucionado en el uso de las TIC durante el año 2011 (Vaillant y Bernasconi, 2013:9).

Al analizar las "percepciones de los docentes sobre el impacto del Plan Ceibal en sus prácticas", los datos obtenidos revelan que "un $59 \%$ se siente inseguro o poco competente" para enfrentar los desafíos que supone la incorporación de las TIC a las prácticas de enseñanza, mientras que "un $4 \%$ se reconoce incompetente". No obstante ello, se vislumbra un impacto positivo en algunos aspectos. "Reflexionar sobre sus prácticas" es el aspecto en el que los docentes perciben que el programa de innovación ha tenido su mayor impacto (señalado por el $78 \%$ de los encuestados), seguido de "tener una actitud expectante frente al mismo" (76 \%). Por su parte, visualizan en una menor proporción el impacto positivo de "profundizar en el uso de las TIC" (69 \%) y de "comprar equipamiento o tener conexión a Internet" (33 \%) (Vaillant y Bernasconi, 2013:10).

Consultados los docentes que participaron del estudio en referencia a su "percepción sobre el cambio en sus prácticas de enseñanza a partir del Plan Ceibal", es posible identificar un grupo de docentes que apuesta al cambio, integrando las TIC a sus prácticas de enseñanza $(32,17 \%)$. Al vincular dichas prácticas con el contexto liceal, los datos indican que "La proporción de profesores en los liceos sin PIU que señalan que sus prácticas han cambiado es el doble que en liceos con PIU4" (Vaillant y Bernasconi, 2013:13). 
Una de las variables que, a juicio del investigador, podrían explicar esa situación, sería que en los liceos ubicados en contextos desfavorables existe menos compromiso con el uso y cuidado del recurso por parte de los alumnos y sus familias. En ese sentido, los profesores de Matemática podrían verse limitados por esta situación, la que afectaría y restringiría las posibilidades de planificar la enseñanza con TIC.

El informe finaliza con algunas conclusiones y recomendaciones. Vaillant y Bernasconi (2013:24-25) advierten sobre la necesidad de atender "la distribución de las capacidades docentes y la innovación con la XO", en lo que respecta a las condiciones institucionales y contextuales; el liceo como unidad de cambio y la fuerza dirigida al cambio, apoyo y sostén de la innovación. Agregan asimismo como factores a considerar, la formación y titulación docente, la promoción del trabajo por proyectos, el contrato docente y la estabilidad en la función.

\section{Metodología}

El estudio priorizó un enfoque de triangulación metodológica, que integró métodos cuantitativos y cualitativos por considerar que ambos paradigmas de investigación son complementarios (Cook y Reichardt, 2000; Pérez Serrano, 2007). Al respecto, Pérez Serrano (2007:10) señala que "El investigador debe ser capaz de entresacar de cada enfoque aquello que más le convenga en orden a una mejor resolución del problema objeto de estudio".

\subsection{Etapa cuantitativa}

Para aproximarnos al real conocimiento de las concepciones docentes sobre el uso de las TIC en las buenas prácticas de enseñanza de la Matemática se propuso, en primera instancia, la realización de una encuesta autoadministrada que incluyó preguntas abiertas y cerradas (Hernández Sampieri et al., 2010).

El cuestionario utilizado estuvo dirigido a los cincuenta y dos docentes de Matemática que se desempeñaban en los liceos públicos de Educación Secundaria del departamento de Artigas, existiendo cuatro apartados bien diferenciados en él: información general, la tarea docente y las TIC, Uso de las TIC en el ámbito personal y profesional y, por último, formación en TIC y valoración del Plan Ceibal. La confiabilidad y validez del instrumento estuvieron dadas por la sistematización y la estandarización de la recolección de datos (Hernández Sampieri et al., 2010), lo que fuera perfeccionado a partir de los pretests realizados. La entrada a campo se realizó luego de contar con el aval institucional del CES y teniendo en cuenta las consideraciones recibidas ${ }^{5}$ (Oficio N . ${ }^{\circ} 376 / 13$ ).

Luego de finalizado el trabajo de campo se procedió a organizar los datos para su posterior análisis. En primer lugar, se realizó la codificación de las variables y posteriormente su tabulación. En un tercer momento, se procedió a la confección de la matriz de datos a través del empleo del paquete estadístico SPSS. En primera instancia, se realizó un análisis descriptivo univariado y, posteriormente, se realizó un análisis bivariado mediante tablas de contingencia.

\subsection{Etapa cualitativa}

Para complementar el análisis y contar con otros elementos que permitieran profundizar en el conocimiento de la relación existente entre las concepciones docentes sobre el uso didáctico de las TIC y las buenas prácticas de la enseñanza de las matemáticas se optó también por un enfoque metodológico cualitativo a partir de un estudio de casos múltiples. Para dicho estudio, cada docente consultado y el centro al cual pertenece en su contexto determinado, constituyen un caso específico. 
En esta etapa, y siguiendo las recomendaciones de Cea D'Ancona (1997:182), se optó por un muestreo intencional no probabilístico ya que, como sostiene, es más apropiado para "profundizar en la información aportada que en una representatividad estadística". Como técnica, se optó por la entrevista en profundidad, ya que la misma permite "la obtención de una gran riqueza de información en las palabras y en los enfoques" (Valles, 1999:201). El trabajo se complementó con el análisis de documentos ${ }^{6}$ (programas de la asignatura, planes anuales, planificaciones, entre otros) y la triangulación metodológica de datos y fuentes (Aravena et al., 2006, Taylor y Bogdan, 1987).

La selección de los entrevistados se realizó a partir de lo manifestado por los agentes que desarrollaban tareas de supervisión educativa, así como también de los profesores de Matemática que se desempeñaban en los liceos públicos del departamento de Artigas.

En el instrumento empleado en la etapa cuantitativa se incluyó un ítem destinado a que los docentes encuestados indicaran directamente a dos compañeros que, según sus percepciones, se destacaran de manera exitosa por desarrollar buenas prácticas de enseñanza de las matemáticas empleando las TIC. En función de ello y de lo señalado por los agentes de supervisión, se seleccionó a cinco docentes para que participaran de esta etapa.

El estudio de los relatos emanados de las diferentes entrevistas en profundidad se llevó a cabo mediante una matriz y un diagrama de análisis, lo que permitió identificar núcleos temáticos en común entre los diferentes discursos, así como también los factores con los que se asociaban. Siguiendo las recomendaciones de Mejía Navarrete (2011) se realizó, en primera instancia, la reducción de datos. Posteriormente, se codificó cada una de las unidades categoriales con una determinada notación. Para dicha tarea se empleó el criterio inductivo-deductivo, partiendo de un sistema de categorías previamente elaborado, el que se fue complementando con las que surgieron a partir del estudio de los diferentes discursos.

Finalmente, se realizó el análisis descriptivo de los datos reducidos y procesados. Se infirieron los enunciados parciales de carácter empírico y descriptivo que permitieron la elaboración del enunciado sintético, insumo relevante para la tarea de interpretación (Mejía Navarrete, 2011).

\section{Discusión de los hallazgos}

En esta sección se busca integrar y contrastar los hallazgos referidos a los profesores de Matemática y sus prácticas de enseñanza con TIC, destacando los aspectos que se consideran más significativos.

\subsection{Planificación de clase con uso de tic}

Una de las tareas inherentes al quehacer docente es la planificación, mediante la cual el profesor toma decisiones referentes a los procesos de enseñanza y de aprendizaje que pretende desarrollar y promover, respectivamente (Gimeno, 1998). Preguntados los docentes de Matemática "¿En promedio, cuántas horas semanales le dedica usted a la planificación del trabajo de aula?", se obtuvo que destinaban, en promedio, 6,4 horas.

Asimismo, se les proporcionó cinco actividades (siendo (1) la primera y (5) la última), para que ordenaran según la prioridad que les atribuyen y de acuerdo a la secuencia que siguen (o seguirían) al momento de planificar una clase con uso de TIC. 
Calculados los promedios de las puntuaciones adjudicadas por los docentes consultados a cada una de las cinco actividades, la secuencia que seguirían, en general, sería la siguiente:

\begin{tabular}{|c|c|c|}
\hline & \multicolumn{2}{|l|}{$\begin{array}{l}\text { TABLA N 1: Profesores de Matemática de Artigas según secuencia que siguen } \\
\text { (o seguirían) al momento de planificar una clase con uso de TIC. }\end{array}$} \\
\hline & Afirmación & Promedio \\
\hline 1 & Reviso el currículum para elaborar mis objetivos de enseñanza. & 2,5 \\
\hline 2 & Formulo los objetivos de la clase. & 2,7 \\
\hline 3 & Evalúo detalladamente los recursos TIC a utilizar. & 2,8 \\
\hline 4 & Verifico los conocimientos previos de los alumnos respecto a los contenidos. & 3,3 \\
\hline 5 & $\begin{array}{l}\text { Verifico los conocimientos previos de los alumnos con respecto a dicha } \\
\text { tecnología. }\end{array}$ & 3,4 \\
\hline
\end{tabular}

Como puede observarse, los docentes consultados en primera instancia revisarían el currículum para elaborar sus objetivos de enseñanza y en función de ello evaluarían y seleccionarían los recursos TIC a utilizar. Por ese motivo, se buscó conocer qué recomendaciones se establecían en el programa vigente de la asignatura en los diferentes niveles de Educación Secundaria referente al uso de las TIC. Del análisis realizado ${ }^{7}$ se concluyó que, desde lo curricular, no existían referencias explícitas que pautaran el trabajo con las TIC en los cursos de Matemática de Ciclo Básico y Bachillerato. Sin embargo, al dialogar de forma informal ${ }^{8}$ con un agente de supervisión educativa para la asignatura a nivel nacional, este indicaba que "se estimula su uso como una herramienta para potenciar aprendizajes significativos que requieran procesos cognitivos complejos" (Inspector de Matemática, 02/08/2013).

\subsection{Usos en el aula que sería importante fomentar}

Las percepciones de los docentes acerca de cuándo sería importante fomentar en los alumnos el uso de las TIC fue otra de las dimensiones exploradas.

Los profesores consultados destacaron que, con mayor frecuencia ${ }^{9}$, era pertinente el uso "para practicar lo trabajado" (77\%), "para poner en funcionamiento un método o concepto aprendido" (71,8 \%) y "para buscar información relevante" (69,2 \%). En el otro extremo, "para no perder tiempo en cálculos o trazados" era la finalidad que registraba la menor adhesión (38,4 \%) de los docentes como un uso que sería importante fomentar. En proporciones intermedias aparecían las otras finalidades consideradas.

Estos datos ratificaban los hallazgos del estudio realizado por la Universidad ORT Uruguay (Vaillant y Bernasconi, 2012) que, con algunos pequeños matices en los porcentajes, ordena de la misma manera los usos presentados, según las percepciones de los profesores de Matemática de primer año del liceo.

Esta situación podría estar advirtiendo sobre una escasa orientación de los docentes hacia la promoción de un trabajo pedagógico en el aula apoyado con tecnología, así como también la ausencia de buenas prácticas de enseñanza de la Matemática mediadas por las TIC. 


\subsection{Usos en el aula que sería importante evitar}

Por una parte, al indagar acerca de cuáles son las situaciones en las que los docentes consideraban que sería pertinente evitar el uso de las TIC en el aula, se observaron niveles bajos de acuerdo en aquellas asociadas a la actividad matemática propiamente dicha: "Aprenden un concepto matemático" registraba el 7,7 \% de las adhesiones, mientras que "Necesitan realizar cálculos numéricos" consignaba el 30,8 \%. Ello estaría reflejando una valoración positiva de los docentes referente a la incorporación de las TIC en el aula para desarrollar procesos de enseñanza y aprendizaje de la asignatura.

Por otra parte, existía un grado de acuerdo alto en lo referente a evitar el uso del recurso cuando los alumnos "Tienen un comportamiento inadecuado en clase", según lo manifestado por tres de cada cuatro docentes consultados. Los siguientes testimonios son un claro exponente de ello:

"en grupos que no he podido usar ha sido porque el grupo no... no... no ayuda, o son chiquilines que no traían las máquinas o no las iban a traer porque no les interesaba, o que cuando estaban trabajando no los podía...viste que a veces es complicado el trabajo en equipo, es complicado ese tipo de cosas" (E1:202-205).

"hay grupos que si no se ha trabajado el... el comportamiento, cuando pones algo así no va a resultar." (E5:248-249) "me parece fundamental lo del comportamiento y la actitud, que se trabaja diariamente para esas instancias. Creo que son donde se reflejan más y donde es más necesario que te escuchen, que estén atentos, que esperen, que vean qué es lo que se quiere, para después empezar a trabajar" (E5:254-257).

Asimismo, el 61,5\% declaraba conveniente evitar su uso en el aula cuando "Existen pocas XO en funcionamiento en la clase", lo que alerta sobre una problemática que debería analizarse en detalle y sobre la cual profundizaremos en el próximo apartado.

\subsection{Perfil de los grupos que los docentes tienen a su cargo y disponibilidad de TIC}

Según datos oficiales del CES, el total de alumnos matriculados en 2013 en el departamento de Artigas era de 6 453, de los cuales 2506 correspondían a Bachillerato y 3947 a Ciclo Básico (CES, 2013b). Se distribuían en once centros, con diferencias en la matrícula según la zona geográfica en la que están ubicados, lo que ocasionaba que los grupos tuvieran una distribución desigual en número de estudiantes.

Un 35,9\% de los profesores consultados indicó menos de 25 alumnos en cada uno de los grupos a su cargo. Se registró un mismo número de docentes con grupos de 26 a 30 alumnos. Un 17,9 \% de los docentes consignó un guarismo de 31 a 35 estudiantes por grupo y sólo un 10,3 \% indicó tener a su cargo grupos de entre 36 y 40 alumnos.

\subsection{Estado de conservación de las computadoras XO pertenecientes a los alumnos}

El promedio de alumnos a cargo de los docentes consultados fue de 134, con una desviación típica de 58 que se fundamentaba en la realidad anteriormente descrita. En la siguiente tabla se presenta la información recabada referente al funcionamiento de las computadoras XO de los estudiantes, según las percepciones de los profesores. 
TABLA N²: Profesores de Matemática de Artigas según funcionamiento de las $\mathrm{XO}$ de los alumnos que tienen a cargo

\begin{tabular}{|c|c|c|}
\hline & $\begin{array}{l}\text { Alumnos que tienen la XO } \\
\text { funcionando }\end{array}$ & $\begin{array}{c}\text { Alumnos que no tienen la } \\
\text { XO funcionando }\end{array}$ \\
\hline Media & 42 & 77 \\
\hline Desviación Típica & 38 & 47 \\
\hline \multicolumn{3}{|c|}{ Fuente: Encuesta aplicada en el marco de la tesis. (Elaboración propia). } \\
\hline
\end{tabular}

Como puede apreciarse, existían diferencias significativas respecto al funcionamiento de las XO de los alumnos que los docentes consultados tenían a cargo.

Al indagar los motivos por los cuales los estudiantes no disponían del recurso funcionando, según lo indicado por los docentes consultados se identificaban los siguientes motivos: la tienen bloqueada $(10,4 \%)$, está rota y la envió a reparar (14,3\%), está rota y no la envió a reparar (24,6 \%), otros motivos (34 \%). De los 3133 estudiantes que concentraban los docentes encuestados (48,5\% del total de la matrícula del departamento), solamente el 19,6 \% tenía el recurso en funcionamiento (613 estudiantes).

Los testimonios recogidos desde el abordaje cualitativo ponían en evidencia nuevos elementos de análisis para esta problemática, al valorar positivamente la presencia del servicio técnico de Plan Ceibal en los liceos.

"en el liceo en el que estoy, permanentemente, hay gente que viene a reparar las máquinas de los jóvenes" (E2:69-70)

"Si no la reparan [los alumnos] es porque no quieren, porque a los liceos van y están un día dedicados a hacer cambios, a reparar computadoras que no están funcionando, que están bloqueadas, como qué... [...] Es gratuito y está bastante seguido, no es que vienen una vez al año" (E5:122-127).

\subsection{Frecuencia de uso y modalidades de trabajo}

Según las declaraciones de los profesores consultados, la frecuencia de uso de las computadoras XO en clase era en promedio de 1,7 horas por semana. Sin embargo, la desviación típica era de 2,6, lo que indicaba que era alta la dispersión de los datos, confirmando que existía un grupo de profesores que no las utilizaba en clase.

Ante la pregunta "Independientemente del número de XO que están en funcionamiento en sus grupos, cuando usted propone una actividad en el aula, en promedio ¿con cuántas XO cuenta para trabajar?", los datos relevados indicaban que en promedio disponían de ocho computadoras (y con una alta desviación típica: 11,5). Esto obligaba a los docentes a idear diferentes modalidades de trabajo para subsanar dicha problemática.

Una de las interrogantes que surgía de inmediato ante el reducido número de equipos con que disponían los docentes para implementar actividades en el aula era “¿Cómo trabajan cuando se realizan actividades en clase con la XO, los alumnos que tienen su equipo roto o en reparación?". Como se puede apreciar en la Tabla N³ 3 el trabajo en equipo era la principal alternativa en ese sentido. Solamente un 10,3 \% de los profesores consultados manifestaba que los alumnos que no disponían del recurso "Trabajan en el cuaderno" (para los grupos de hasta 25 alumnos, dos tercios se hallaban en esta situación). 


\begin{tabular}{|c|c|}
\hline \multicolumn{2}{|c|}{$\begin{array}{l}\text { TABLA N 3: Profesores de Matemática de Artigas según modalidad de trabajo que } \\
\text { implementan para aquellos alumnos que no tienen su XO en funcionamiento }\end{array}$} \\
\hline Afirmación & $\%$ \\
\hline Trabajan en equipos con alumnos que sí las tienen. & 51,3 \\
\hline Usan las XO de alumnos de otras clases. & 33,3 \\
\hline Usan las XO del Laboratorio de Informática del Liceo. & 25,6 \\
\hline Trabajan en el cuaderno. & 10,3 \\
\hline Fuente: Encuesta aplicada en el marco d & \\
\hline
\end{tabular}

\subsection{La inclusión de las TIC en las prácticas de enseñanza}

Ante la pregunta "¿Usted incluye la XO en sus prácticas de enseñanza?", el 56 \% de los docentes consultados respondía afirmativamente. Como puede apreciarse, existía un número importante de profesores (17 de 39) que declaraba no desarrollar aún prácticas de enseñanza de la Matemática mediadas por las TIC.

Con el objetivo de describir con mayor precisión las características de ambos grupos de profesores (los que las incluyen y los que no), se profundizó en el análisis a partir de diferentes cruces entre variables buscando establecer si existían asociaciones o no entre las mismas.

\begin{tabular}{|c|c|c|c|}
\hline \begin{tabular}{|c|c|}
\hline TABLA N \\
4: Inclusión de las XO en las prácticas de enseñanza según titulación como \\
profesor de Matemática
\end{tabular} \\
\hline \multicolumn{4}{|c|}{ PROFESOR DE MATEMÁTICA } \\
\hline INCLUYE LAS XO & Titulado & No titulado & TOTAL \\
\hline Sí & $63 \%$ & $55 \%$ & $56 \%$ \\
\hline No & $37 \%$ & $45 \%$ & $44 \%$ \\
\hline TOTAL & $100 \%$ & $100 \%$ & $100 \%$ \\
\hline Fuente: Encuesta aplicada en el marco de la tesis. (Elaboración propia). \\
\hline
\end{tabular}

Como se puede apreciar en la tabla anterior, el porcentaje de docentes que indicaba que había integrado las XO a sus prácticas de enseñanza era levemente superior entre aquellos docentes que poseían titulación específica como Profesor de Matemática para Enseñanza Media. Los datos mostraban que solamente tres de cada diez docentes titulados consultados no incluían las XO a sus prácticas de enseñanza, mientras que la relación era de cuatro en diez entre los docentes no titulados.

Sin embargo, al confrontar los datos relativos a la inclusión de las XO en las prácticas de enseñanza con el grado que poseía el profesor (Ver Tabla N5), se observaban diferencias significativas en algunos casos. Los docentes consultados que indicaron estar ubicados en grado 1, 3 o 7, se distribuían con igual proporción entre los dos grupos. Por otra parte, para los docentes de grado 2 , se observaba una sensible diferencia entre los que las incluían frente a los que no lo realizaban. No obstante ello, los docentes de grado 4 y 5 no las empleaban en absoluto, mientras que los de grado 6 las utilizaban en su totalidad. 
TABLA N 5: Inclusión de las XO en las prácticas de enseñanza según grado del profesor

\begin{tabular}{|c|c|c|c|c|c|c|c|c|}
\hline \multicolumn{10}{|c|}{ GRADO } \\
\hline INCLUYE XO & 1 & 2 & 3 & 4 & 5 & 6 & 7 & TOTAL \\
\hline Sí & $54 \%$ & $83 \%$ & $50 \%$ & $0 \%$ & $0 \%$ & $100 \%$ & $50 \%$ & $56 \%$ \\
\hline No & $46 \%$ & $17 \%$ & $50 \%$ & $100 \%$ & $100 \%$ & $0 \%$ & $50 \%$ & $44 \%$ \\
\hline TOTAL & $100 \%$ & $100 \%$ & $100 \%$ & $100 \%$ & $100 \%$ & $100 \%$ & $100 \%$ & $100 \%$ \\
\hline
\end{tabular}

Fuente: Encuesta aplicada en el marco de la tesis. (Elaboración propia).

Por otra parte, fue relevante considerar el ciclo en el que trabajaba el profesor con el propósito de analizar si existían diferencias significativas en ese sentido. Como se puede visualizar en la Tabla $N^{\circ} 6$, existía una fuerte asociación entre el ciclo y la inclusión de las XO en las prácticas de enseñanza. Mientras que siete de cada diez docentes que se desempeñaban en Ciclo Básico declaraban integrar las XO en sus clases, en Bachillerato se mantenía la proporción inversa.

TABLA Nº 6: Inclusión de las XO en las prácticas de enseñanza según ciclo en el que trabaja el profesor

\begin{tabular}{|c|c|c|c|c|}
\hline \multicolumn{5}{|c|}{ CICLO EN EL QUE TRABAJA } \\
\hline INCLUYE XO & Ciclo Básico & Bachillerato & Ambos & TOTAL \\
\hline Sí & $74 \%$ & $29 \%$ & $66 \%$ & $56 \%$ \\
\hline No & $26 \%$ & $71 \%$ & $34 \%$ & $44 \%$ \\
\hline TOTAL & $100 \%$ & $100 \%$ & $100 \%$ & $100 \%$ \\
\hline
\end{tabular}

Finalmente, en la Tabla $N^{\circ} 7$ se presenta la relación entre la inclusión de las XO en las prácticas de enseñanza y la percepción de los docentes consultados sobre su evolución en el desempeño de uso de las TIC durante el año 2013. Al comparar las diferencias porcentuales entre las categorías establecidas, se constataba que las percepciones positivas sobre la evolución personal en el desempeño de uso de las TIC favorecían su integración a las prácticas de enseñanza.

\section{TABLA N ${ }^{\circ}$ 7: Inclusión de las XO en las prácticas de enseñanza según percepción de} evolución en el desempeño de uso de las TIC durante el año 2013

\begin{tabular}{|c|c|c|c|c|c|}
\hline \multicolumn{5}{|c|}{ EVOLUCIÓN DEL DESEMPEÑO TIC } \\
\hline INCLUYE XO & Nada & Poco & Bastante & Mucho & TOTAL \\
\hline Sí & $34 \%$ & $47 \%$ & $72 \%$ & $50 \%$ & $56 \%$ \\
\hline No & $66 \%$ & $53 \%$ & $28 \%$ & $50 \%$ & $44 \%$ \\
\hline TOTAL & $100 \%$ & $100 \%$ & $100 \%$ & $100 \%$ & $100 \%$ \\
\hline
\end{tabular}

En lo referente al grado de incorporación de las XO a diferentes aspectos del quehacer docente, las percepciones de los profesores de Matemática evidenciaban una situación alarmante. Según el índice creado ${ }^{10}$, asumían valores positivos únicamente dos de los doce aspectos puestos a consideración: "La búsqueda de actividades motivadoras" $(20,6$ \%) y "El desarrollo de propuestas áulicas" (2,5\%). 


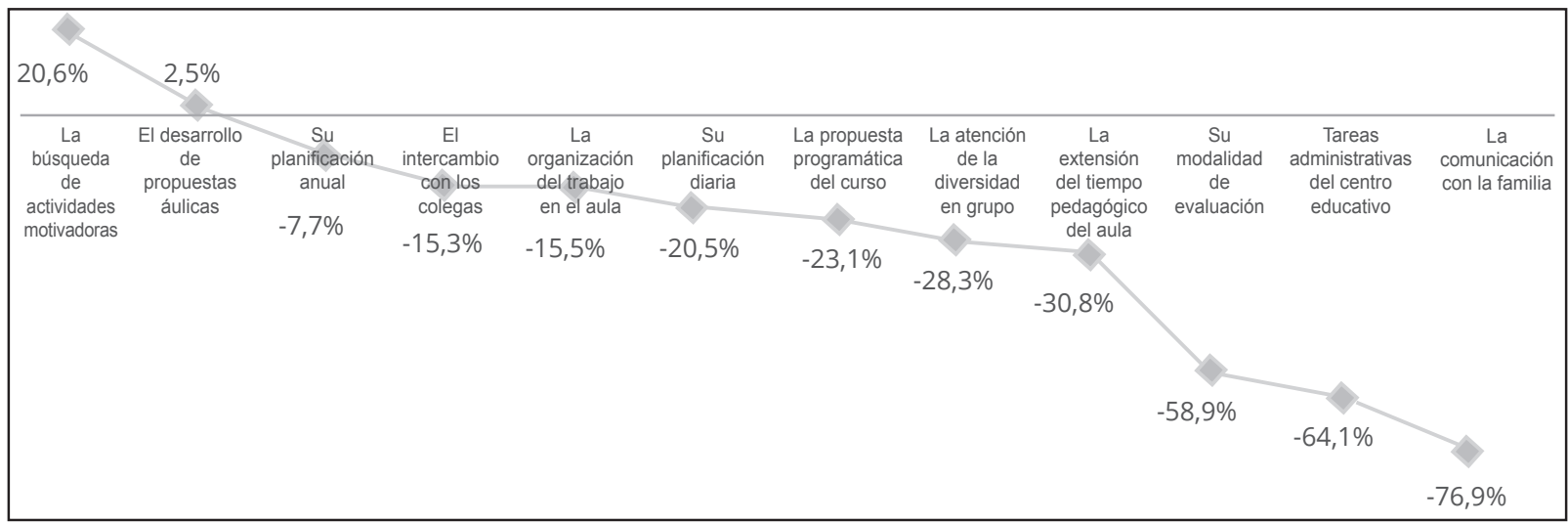

Fuente: Encuesta aplicada en el marco de la tesis (Elaboración propia).

\section{Gráfico 1. Profesores de Matemática de Artigas según su autopercepción acerca de en qué han logrado integrar las XO en diferentes aspectos}

Tal como puede apreciarse en el gráfico anterior, las principales dificultades constatadas referían a: “La comunicación con la familia” (-76,9\%), la realización de “Tareas administrativas del centro educativo" (-64,1 \%) y la "Modalidad de evaluación" (-58,9\%). Sin lugar a dudas, estas tareas requieren un mayor compromiso del docente con la innovación y su adhesión a un nuevo paradigma educativo, cuyo enfoque está muy distante de la realidad que se percibía en las aulas de los liceos de Educación Secundaria del departamento de Artigas.

Asimismo, se consultó a los profesores acerca de si habían cambiado sus prácticas de enseñanza a partir del uso de la XO. El 51,3 \% indicaba que sí, mientras que el 30,8 \% señalaba que no. El porcentaje de profesores que no respondían ascendía a 17,9 \%.

La realidad constatada pone en evidencia la imperiosa necesidad de contar con una política de formación docente en TIC, que fomente el desarrollo de las competencias y habilidades necesarias para lograr ofrecer "experiencias de aprendizaje pertinentes, enriquecidas, atractivas y desafiantes a los aprendices del siglo XXI" (Unesco, 2013:38).

\subsection{Impacto de las TIC en las prácticas de enseñanza}

La encuesta exploró las percepciones de los profesores acerca de los resultados obtenidos en diferentes dimensiones a partir de la incorporación de las TIC a sus prácticas de enseñanza. Se solicitó a los docentes que valoraran el impacto observado en cada una de ellas de acuerdo con una escala de cuatro categorías. Para facilitar el análisis se creó un índice-resumen que resultó de la suma de los porcentajes atribuidos a las categorías "Bastante" o "Mucho", y de la resta de los porcentajes de las categorías "Nada", "Poco" y "No contesta", cuyos valores pueden apreciarse en el siguiente gráfico: 


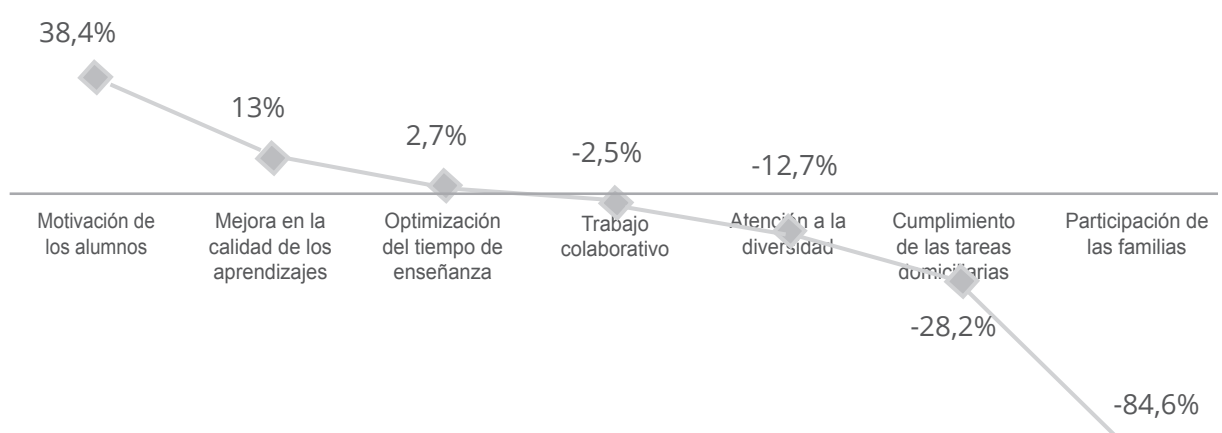

Fuente: Encuesta aplicada en el marco de la tesis (Elaboración propia).

\section{Gráfico 2. Profesores de Matemática de Artigas según su percepciones sobre resultados obtenidos a partir de la incorporación de las TIC a sus prácticas de enseñanza en el aula.}

Como podemos observar, el índice creado arrojó un resultado positivo del 38,4 \% asociado a la motivación de los alumnos, mientras que en menor proporción (13\%) se situaba el impacto vinculado a la mejora en la calidad de los aprendizajes.

Los siguientes testimonios de dos docentes entrevistadas son muy elocuentes en ese sentido.

“cuando se usó la máquina fueron sorprendentes los resultados, es decir, cómo chiquilines que uno los veía como... con la participación muy tenue, cuando uno les propone tareas en... con la máquina enfrente, los resultados son distintos" (E2:100-102).

"que todos los alumnos cuando vos ponés un trabajo para que hagan en la computadora, se enganchan, hacen, o por lo menos intentan. Que les salga o no, es otra cosa, pero como que hay esa disposición que quizá en otro momento no hay" (E5:241-244).

Por otra parte, el índice indicaba que no se visualizaban aportes significativos en la participación de la familia (-84,6\%) y en el cumplimiento de las tareas domiciliarias (-28,2 $\%$ ), situación que estaría advirtiendo sobre el hecho de que el programa de innovación aún no ha logrado consolidar redes de comunicación que traspasen las "barreras" del aula. Un reciente estudio de Unesco indica que: “Las tecnologías facilitan las redes de comunicación y permiten mejorar el vínculo de la familia, la escuela, los estudiantes y otros organismos locales" (Unesco, 2013:37).

\section{Conclusiones}

En el presente capítulo se presentan las conclusiones más destacadas, que surgen del análisis de la información recabada en el marco del trabajo de campo realizado.

Al indagar acerca de las percepciones de los profesores de Matemática sobre sus prácticas de enseñanza con TIC, se constató que, en promedio, los profesores dedicaban 6,4 horas a la planificación de la labor docente de forma semanal, representando dicho guarismo el $12 \%$ de la carga horaria semanal que, en promedio, tienen los docentes consultados. 
Los profesores encuestados consideraban importante fomentar el uso de las TIC para tareas específicas como "trabajar lo practicado", "poner en funcionamiento un método o concepto aprendido" y "buscar información relevante". Sin embargo, el ítem "para no perder tiempo en cálculos o trazados" aparecía como el uso menos sugerido por los docentes. En lo que refiere a los usos pedagógicos que sería importante evitar, aparecieron de forma jerarquizada aquellas situaciones particulares en las que los alumnos "tienen un comportamiento inadecuado en clase".

El estado de conservación y disposición de las computadoras es una problemática que suscitó la atención de los profesores consultados. Considerando la cantidad de alumnos por grupo, el número de computadoras XO disponibles era muy bajo, tal como lo indicaba la alta dispersión de los datos. Sin embargo, los testimonios recogidos desde el abordaje cualitativo confirmaban la presencia sistemática y periódica del servicio técnico de Plan Ceibal, abocado a la tarea de reparación y mantenimiento de los equipos.

La frecuencia de uso de las TIC en las prácticas de enseñanza de los docentes consultados era de 1,7 horas por semana, dentro de una carga horaria semanal promedio de 48 horas de trabajo. Al igual que otros estudios nacionales, la evidencia reveló que los profesores de Matemática incluían muy poco el recurso en sus prácticas de enseñanza. Confirmando esta idea, la alta dispersión de los datos indicó que existía un grupo de profesores que no las utilizaba en sus clases, lo que se corroboraba desde los testimonios que surgían de las entrevistas. En promedio, los profesores disponían de ocho computadoras por clase, lo que obligaba a diseñar alternativas para poder gestionar la enseñanza mediada por las TIC: el trabajo en equipo era la estrategia seleccionada por excelencia.

Las percepciones sobre la inclusión de las TIC y los cambios en las prácticas de enseñanza a través de los usos que de las mismas se realizaba, indicaban una fuerte asociación con respecto al ciclo en el que se desempeñaba el profesor: siete de cada diez docentes las incluía en Ciclo Básico, mientras que la realidad de Bachillerato se mantenía en proporción inversa. Con menor intensidad, se observaban diferencias en la inclusión de las TIC según la titulación docente: siete de cada diez docentes titulados las incluía en sus prácticas, mientras que para los no titulados la relación era de seis a diez. La investigación también concluyó que las percepciones positivas sobre la evolución personal en el desempeño del uso de las TIC favorecían su integración a las prácticas de enseñanza.

Entre los principales hallazgos de esta investigación se destacó una contradicción entre lo que los docentes piensan (sus concepciones y opiniones) y lo que declaran hacer (sus prácticas). Presentan una visión positiva sobre el uso de las TIC en las prácticas de enseñanza y señalan aspectos vinculados a diferentes dimensiones del quehacer docente que hacen a una buena práctica de enseñanza de la Matemática. Sin embargo, se constató que en general no las integraban y existía un escaso uso didáctico de las mismas. 


\section{Referencias bibliográficas}

Aravana, M., Kimelman, E., Micheli, B. Torrealba, R. Zúñiga, J. (2006). Investigación Educativa I. Chile: Universidad ARCIS.

Calvo, I. \& Gil, L. (2013). Formación de docentes de Matemática en el uso de recursos tecnológicos. En Actas del VII CIBEM, 536 - 547. Montevideo: SEMUR.

Castillo, S. (2008). Propuesta pedagógica basada en el constructivismo para el uso óptimo de las TIC en la enseñanza y el aprendizaje de la Matemática. Revista Latinoamericana de Investigación en Matemática Educativa, 11(2), 171-194. Recuperado de: http://www.scielo. org.mx/scielo.php?pid=S1665-24362008000200002\&script=sci_arttext

Cea D’Ancona, M. (1997). Metodología cuantitativa: estrategias y técnicas de Investigación Social. Madrid: Síntesis.

CEIBAL. (2011b). Módulo 2: Modelo educativo 1 a 1 como propuesta de innovación educativa. Versión Digital (PDF) En: Curso escenarios educativos con dotación 1:1. Construyendo el conocimiento desde la práctica.

Ceibal. (2011c). Encuesta a docentes de educación media pública sobre acceso, dominio y uso de herramientas TIC. Montevideo: Departamento de Monitoreo y Evaluación del Plan Ceibal. Recuperado de http://www.ceibal.edu.uy/Documents/ENCUESTA\%20A\%20 DOCENTES\%20DE\%20EDUCACI\%C3\%93N\%20MEDIA\%20P\%C3\%9ABLICA\%20SOBRE\%20 ACCESO,\%20DOMINIO\%20Y\%20USO\%20DE\%20HERRAMIENTAS\%20TIC\%202011.pdf

CES. (2013a). Oficio № 376/13. Montevideo: CES. Accedido el 30 de julio, 2013, desde http:// www.ces.edu.uy/ces/index.php?option=com_content\&view=article\&id=6568:exp714713oficio-no37613m/p\&catid=174:oficio

CES. (2013b). Categorización de liceos para año 2014 - Datos matrícula 2013. Montevideo: CES. Recuperado el 20 de noviembre de 2013, de: http://www.ces.edu.uy/ces/images/ stories/efimeros/recategorizacion/recategorizaci\%C3\%B3n.pdf

Chevallard, Y. (1991). La transposición didáctica: del saber sabio al saber enseñado. Buenos Aires: Aique.

Chevallard, Y., Bosch, M. \& Gascón, J. (1999). Estudiar Matemáticas. El eslabón perdido entre la enseñanza y el aprendizaje. Barcelona: Horsori.

Cook, T. \& Reichardt, Ch. (2000). Métodos cualitativos y cuantitativos en investigación evaluativa. Madrid: Morata.

De Pablos Pons, J. \& González Ramírez, T. (2007). Políticas educativas e innovación educativa apoyada en TIC: sus desarrollos en el ámbito autonómico. Recuperado de http:// redes-cepalcala.org/inspector/DOCUMENTOS\%20Y\%20LIBROS/TIC/GRANADA\%20TIC\%20 2007/1101C.pdf

Flick, U. (2004). Introducción a la investigación cualitativa. Madrid: Morata.

Gimeno, J. (1998). Didáctica y currículo. Barcelona: Morata. 
Hernández Sampieri, R. et al. (2010). Metodología de la investigación. Quinta Edición. Perú: McGraw-Hill.

López-Vargas, B. I. \& Basto-Torrado, S.P. (2010). Desde las teorías implícitas a la docencia como práctica reflexiva. Educación y Educadores, 13(2), 275-291. doi:10.5294/ edu.2010.13.2.6

López Esteban, C. (2011). Mejores Prácticas en la Enseñanza de las Matemáticas: La integración de las TICS. SCOPEO, El Observatorio de la Formación en Red. Boletín Nº 34 Salamanca: España. Recuperado de http://scopeo.usal.es/enfoque-bol-34-mejorespracticas-en-la-ensenanza-de-las-matematicas-la-integracion-de-las-tics/

Lugo, M.T. \& Kelly, V. (2010). Tecnología en educación ¿políticas para la innovación? (1ª ed.). Buenos Aires: Instituto Internacional de Planeamiento de la Educación IIPE-Unesco. Recuperado de http://www.buenosaires.iipe.unesco.org/sites/default/files/Documento\% 2520Tecnologia\%2520en\%2520educ\%2520Lugo\%2520Kelly.pdf

Mejía Navarrete, J. (2011). Problemas centrales del análisis de datos cualitativos. Revista Latinoamericana de Metodología de la Investigación, 1(1), 47-59. Recuperado de http:// www.relmis.com.ar/ojs/index.php/relmis/article/view/11/1

Parra, C. \& Sainz, I. (Comps.). (1994). Didáctica de Matemática: aportes y reflexiones. Buenos Aires: Paidós.

Pérez Gomar, G. (Coord.) \& Ravela, P. (2012). Impactos del Plan Ceibal en las prácticas de enseñanza en las aulas de Primaria. Informe Final. Recuperado de http://www.ibertic. org/evaluacion/sites/default/files/biblioteca/26_impactos_plan_ceibal_ensenanza_aulas_ primaria.pdf

Pérez Serrano, G. (2007). Desafíos de la investigación cualitativa. Chile: Cátedra de Pedagogía Social. Universidad Nacional de Educación a Distancia (UNED). Recuperado de http://www. rmm.cl/usuarios/pponce/doc/200711151514230.6conferencia_gloria_perez_serrano.pdf

Podestá, P. (Comp.). (2011). Geometría (1ª ed.). Buenos Aires: Ministerio de Educación de la Nación. Recuperado de http://bibliotecadigital.educ.ar/uploads/contents/M-Geometria0. pdf

Pozo, J. I. (2000). Aprendices y maestros. Primera Reimpresión. Madrid: Alianza Editorial.

Pozo, J. I. et al. (2006). Nuevas formas de pensar la enseñanza y el aprendizaje. Las concepciones de profesores y alumnos. Barcelona: Graó.

Rivoir, A. L. (2009). Innovación para la inclusión digital. El Plan Ceibal en Uruguay. Mediaciones Sociales. Revista de Ciencias Sociales y de la Comunicación, 1(4), 299-328.

Rodríguez Zidán, E. (Coord.). (2009). Estudio exploratorio sobre el impacto del Plan Ceibal en Salto. Paysandú: Redicor S.A.

Rodríguez Zidán, E. \& Téliz, F. (2013). El Plan CEIBAL, los Profesores de Matemática y sus prácticas con TIC. Revisión de antecedentes de investigación, políticas de mejora y desafíos pendientes. Cuadernos de Investigación Educativa. 4 (19), 13-36. 
Sosa, M. J.; Peligros, S. \& Díaz Muriel, D. (2010). Buenas prácticas organizativas para la integración de las TIC en el sistema educativo extremeño. Teoría de la Educación. Educación y Cultura en la Sociedad de la Información, 11(3), 148-179. Recuperado de http://www. redalyc.org/articulo.oa?id=201014897007

Taylor, S. \& Bogdan, R. (1987). Introducción a los métodos cualitativos de investigación. Buenos Aires: Paidós.

Teds-M. (2012). Estudio internacional sobre la formación inicial en matemáticas de los maestros. Informe Español. Recuperado de http://www.mecd.gob.es/dctm/inee/ internacional/teds-mlinea.pdf?documentld=0901e72b8143866e

Tejedor, F. J., García-Valcácel, A. \& Prada, S. (2009). Medida de actitudes del profesorado universitario hacia la integración de las TIC. Comunicar, XVII (33), 115-124. Recuperado de http://eprints.rclis.org/17595/1/c33-2009-03-002.pdf

UNESCO. (2013). Enfoques estratégicos sobre las TICS en educación en América Latina y el Caribe. Recuperado de http://www.unesco.org/new/fileadmin/MULTIMEDIA/FIELD/ Santiago/pdf/TICS-enfoques-estrategicos-sobre-TICs-ESP.pdf

Vaillant, D. \& Bernasconi G. (2013). El Plan Ceibal y las prácticas de los docentes de Matemática en primer año de liceo. Recuperado de http://ie.ort.edu.uy/innovaportal/ file/10212/1/plan_ceibal_practicas_de_docentes_de_matematica_2013.pdf

Valles, M. (1999). Técnicas cualitativas de investigación social. Reflexión metodológica y práctica profesional. Madrid: Síntesis.

\section{(Endnotes)}

1) Desarrollado en extenso en el trabajo de De Pablos y González (2007).

2) Que según Castillo (2008:14), en Matemática, requiere comprender qué saben los estudiantes y qué necesitan aprender. A partir de ello, hay que retarlos y apoyarlos para que logren una buena formación.

3) Censo Docente del año 1995 (ANEP, 1996). Estudio sobre la enseñanza y el aprendizaje de la matemática en el Primer Ciclo de Educación Media (ANEP-MEMFOD, 2007) y Censo Docente 2007 (ANEP-DIEE, 2007).

4) PIU: Programa de Impulso a la Universalización del Ciclo Básico.

${ }^{5)}$ Referidas a que se debería coordinar con las Direcciones Liceales la visita a los liceos sin entorpecer el normal desarrollo de los cursos. (CES, 2013a).

6) El análisis documental realizado no proporcionó información relevante para la investigación. Se analizaron las planificaciones anuales de dos de los cinco docentes entrevistados, además de los programas vigentes de la asignatura.

7) Consistió en una revisión de los programas vigentes de la asignatura (Reformulación 2006-Ajustes 2010) para todos los cursos de Matemática de Educación Secundaria (de primero de Ciclo Básico a tercero de Bachillerato).

8) Como una entrevista episódica en términos de Flick (2004).

9) Sumando los porcentajes de las categorías "Frecuentemente" y "Siempre".

10) Esto surge se sumar los porcentajes atribuidos a las categorías "Se ha integrado bastante" $y$ "Se ha integrado plenamente", y restar la suma de los porcentajes correspondientes a las categorías "Se ha integrado poco" y "Aún no se ha integrado". 